\title{
Depresión y autopercepción de la carga del cuidado en pacientes en hemodiálisis y sus cuidadores ${ }^{1}$
}

\author{
María Cecilia Arechabala, ${ }^{2}$ María Isabel Catoni, ${ }^{2}$ Eugenia Palma \\ y Silvia Barrios ${ }^{2}$
}

Forma de citar

Arechabala MC, Catoni MI, Palma E, Barrios S. Depresión y autopercepción de la carga del cuidado en pacientes en hemodiálisis y sus cuidadores. Rev Panam Salud Publica. 2011;30(1):74-9.

RESUMEN Objetivo. Determinar sintomas depresivos, apoyo social percibido y autopercepción de carga en pacientes en hemodiálisis crónica (HDC); identificar síntomas depresivos y grado de fatiga en cuidadores de pacientes en HDC, y evaluar —en el paciente - la asociación entre autopercepción de carga y apoyo social percibido, y entre autopercepción de carga y síntomas depresivos, y - en el cuidador - la relación entre el número de años de cuidado y el grado de fatiga alcanzado.

Métodos. Se tomó una muestra de 162 díadas paciente-cuidador, a quienes se les aplicaron los instrumentos "escala adaptada de percepción de apoyo social", "escala de autopercepción de carga", "escala de depresión del Centro de Estudios Epidemiológicos" y "escala de severidad de la fatiga". Los instrumentos fueron aplicados a los pacientes por ayudantes de investigación entrenados y, en el caso de los cuidadores, fueron autoaplicados.

Resultados. Del total de pacientes, $40 \%$ fueron mujeres, con un promedio de edad de $56 \pm$ 14,9 años, mientras que entre los cuidadores principales, 75\% fueron mujeres, cónyuges, con una edad promedio de $50 \pm 16,1$ años. Se observó que tanto los pacientes $(40,74 \%)$ como los cuidadores principales $(43,82 \%)$ presentaban sintomas de depresión. Se encontró una asociación positiva $(r=0,43, \mathrm{P}<0,001)$ entre la autopercepción de carga y la presencia de síntomas depresivos en los pacientes.

Conclusiones. Los cuidadores principales son en su mayoría mujeres y familiares de los pacientes. Se perciben fatigados con el cuidado, al igual que lo perciben los propios pacientes. La presencia de sintomas depresivos es similar en pacientes y cuidadores, y hay una asociación estadísticamente significativa entre autopercepción de carga y presencia de sintomas depresivos en los pacientes.

Palabras clave Hemodiálisis; síntomas depresivos; enfermedad crónica; salud mental; Chile.

El diagnóstico de una enfermedad crónica a una persona tiene repercusiones en su grupo familiar que incluyen

\footnotetext{
Este trabajo fue financiado por la Dirección de Investigación de la Escuela de Enfermería, Pontificia Universidad Católica de Chile.

2 Escuela de Enfermería, Pontificia Universidad Católica de Chile, Santiago, Chile. La correspondencia se debe dirigir a María Cecilia Arechabala. Correo electrónico: marechab@uc.cl
}

problemas económicos, alteraciones de los roles y cansancio de algunos de sus integrantes (1). En el caso de la familia de un paciente con insuficiencia renal crónica terminal (IRCT), sus miembros deben asumir funciones extraordinarias como resultado del tratamiento de sustitución renal al que debe ser sometido, tanto en el hogar como en el centro de diálisis (2).
Hacerse cargo del cuidado de un familiar enfermo implica para quien lo otorga, entre otras complicaciones, descuidar de cierta manera su salud, aislarse o disminuir sus actividades sociales y aumentar su carga de trabajo, así como sufrir ciertas restricciones, consecuencias económicas negativas, cambios en la relación con el paciente y problemas sexuales (3). Más aún, si este cuidado se mantiene por un 
período prolongado de tiempo puede tener consecuencias en la salud de los cuidadores, quienes suelen presentar cuadros tales como fatiga, síntomas depresivos o directamente depresión $(2,4)$.

Si bien quienes atienden a los familiares enfermos generalmente son descritos por los pacientes como un gran apoyo, hay estudios en los que emergen aspectos negativos de esta relación. De hecho, a los enfermos les preocupa la dependencia de otros para realizar sus actividades y perciben, en algunas ocasiones, a sus cuidadores como incapaces de brindar apoyo y poco comprensivos, lo cual se convierte en angustia para relacionarse, fuente de dificultades y resentimientos en el paciente (5). En este sentido, investigaciones que han medido las repercusiones de la enfermedad crónica en quienes la padecen, han encontrado que el hecho de que estas personas perciban un bajo apoyo por parte de sus familiares o amigos incrementa las cifras de morbilidad y mortalidad en este grupo de pacientes (6). Asimismo, la presencia de signos de depresión en las personas con enfermedades crónicas tiene como resultado una disminución en su sobrevida y una importante repercusión en la adherencia al tratamiento tanto farmacológico como no farmacológico (6).

Conocer la percepción de los pacientes respecto a la carga que su cuidado significa para sus cuidadores (autopercepción de carga del paciente) permite identificar grados de estrés en los enfermos y conocer además aspectos importantes de la relación paciente-cuidador, permitiendo así a los profesionales de la salud planificar e implementar intervenciones específicas dirigidas a esta díada (7).

El presente estudio tiene como objetivos determinar síntomas depresivos, apoyo social percibido y autopercepción de carga en pacientes en hemodiálisis crónica (HDC); identificar síntomas depresivos y grado de fatiga en cuidadores de pacientes en HDC, y evaluar - en el paciente- la asociación entre autopercepción de carga y apoyo social percibido, y entre autopercepción de carga y síntomas depresivos, y - en el cuidador- la relación entre el número de años de cuidado y el grado de fatiga alcanzado.

\section{MATERIALES Y MÉTODOS}

El presente estudio es de carácter descriptivo y correlacional. Al momento de su inicio, según el registro de la XXIII
Cuenta de HDC en Chile, en la ciudad de Santiago había 4259 pacientes sometidos a HDC en hospitales y centros privados de diálisis (8), cifra que representa la mitad de la población en HDC de todo el país. Dado que Santiago abarca una extensa superficie donde las características de los pacientes y de los centros de diálisis (CD) podrían no ser homogéneas, se acordó dividir la ciudad en cinco zonas -norte, sur, este, oeste y centro- y seleccionar un $C D$ para que representara a cada una de ellas.

Se aplicaron los criterios de participación en el estudio a la totalidad de los pacientes de estos $5 \mathrm{CD}$, teniendo la precaución de incluir a usuarios de todos los turnos en razón de que esta característica es relevante para la independencia de los pacientes (p. ej. la mayoría de los pacientes del último turno trabaja, a diferencia del resto). La muestra se reunió en abril de 2006 y los criterios de inclusión de la misma se presentan a continuación.

En el caso de los pacientes:

- Haber estado sometido a HDC por un período igual o superior a 3 meses.

- Reconocer a un cuidador principal

- No presentar deterioro cognitivo (evaluado a través del mini-examen del estado mental de Folstein).

- Aceptar voluntariamente participar en el estudio mediante la firma de un consentimiento informado.

En el caso de los cuidadores principales:

- Ser reconocido como cuidador principal por el paciente.

- Aceptar voluntariamente participar en el estudio mediante la firma de un consentimiento informado.

Para fines del estudio, las variables se definieron de la siguiente manera:

- Apoyo social percibido: los aspectos psicosociales y la ayuda tangible que provee la red social y que recibe la persona, cuya verdadera naturaleza o medición hay que buscarla en los procesos perceptivos de los sujetos implicados $(9,10)$.

- Carga del cuidador: sentimientos negativos y experiencia de presión como resultado de cuidar a una persona con enfermedad crónica (7).

- Cuidador principal: persona de la familia o cercana al paciente que se ocupa de darle apoyo físico y/o emocional en forma permanente $\mathrm{y}$ comprometida (11).
FIGURA 1. Conformación de la muestra para estudiar depresión y autopercepción de carga por parte de pacientes en hemodiálisis y de sus cuidadores, Chile, 2006

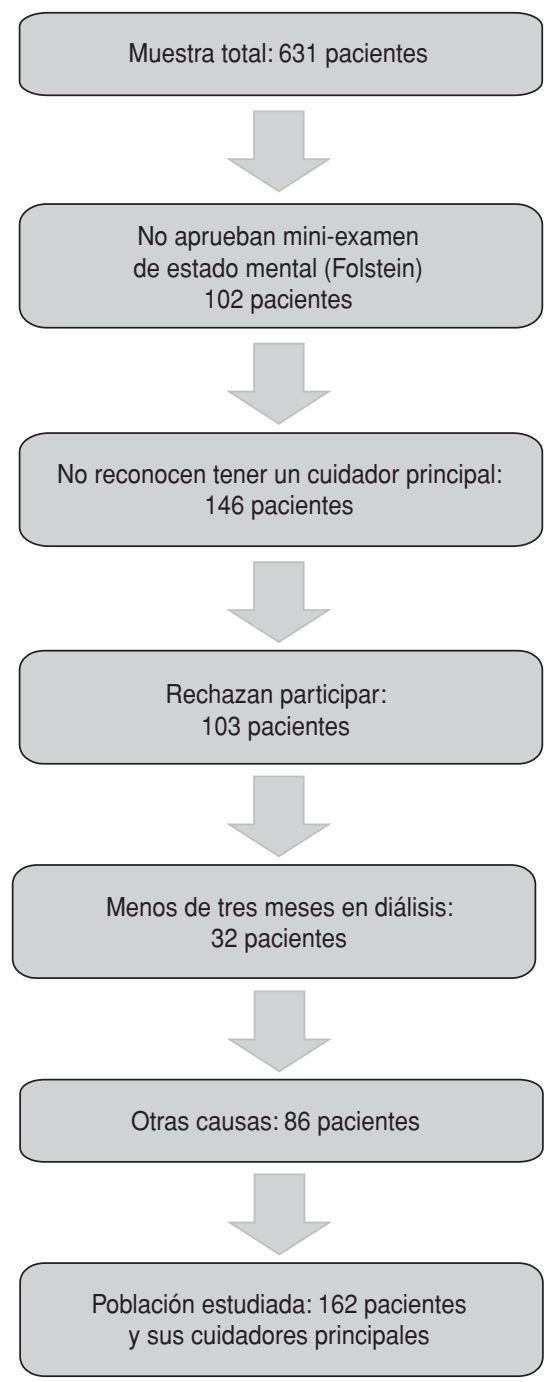

Fuente: elaboración de las autoras.

Después de aplicar los criterios de inclusión al total de 631 pacientes que integraban los $5 \mathrm{CD}$, la muestra quedó constituida por 162 pacientes y sus cuidadores principales (figura 1).

\section{Materiales utilizados para recolectar la información}

\section{Instrumentos aplicados a los pacientes en HDC:}

- Con el objeto de conocer la percepción de apoyo de los pacientes, se aplicó la "escala adaptada de percepción de apoyo social" de Zimet y colaboradores, la cual mide el grado en que el in- 
dividuo percibe el apoyo social de su familia, sus amigos y otros significativos (12). Esta escala fue traducida al español y validada por Arechabala y Miranda, mostrando un alpha de Crombach de 0,88 (13). La versión validada, a diferencia de la original, incluye solamente dos dimensiones: familia y otros significativos. Sus resultados se interpretan adjudicando un mayor puntaje a una mayor percepción de apoyo social, con un puntaje mínimo posible de 12 y un máximo de 60 . Dado que se trata de una escala de puntaje continuo, para fines de interpretación de los resultados se aplicó como punto de corte el valor del promedio obtenido por la muestra más una desviación estándar (DE), lo que significa que los pacientes con un puntaje mayor o igual a 46 puntos perciben un alto apoyo social. Por el contrario, los pacientes que perciben bajo apoyo social serían aquellos con puntaje menor al promedio menos una DE (30,6 puntos). En el presente estudio el alpha de Crombach de este instrumento fue de 0,747 , es decir menor a la versión validada.

- El segundo instrumento aplicado a los pacientes fue la "escala de autopercepción de carga" de Cousineau y colaboradores, cuyo propósito es identificar a pacientes con problemas emocionales debidos a sentimientos de representar una carga para su cuidador (7). Esta escala tiene dos versiones en inglés: una de 25 ítems que ha demostrado una buena consistencia interna (alpha de Crombach de 0,92), y una abreviada de 10 ítems con un alpha de Crombach de 0,85. En la presente investigación se procedió a traducir la versión larga, obteniéndose un alpha de Crombach de 0,90. A efectos de la interpretación de los resultados, los puntajes se convierten a través de una fórmula a puntaje normal, donde el mínimo es cero y el máximo 100, con una relación directa entre el puntaje y la percepción del paciente respecto de la carga que significa — su cuidado - para su cuidador principal (7). Dado que se trata de una escala de puntaje continuo, para fines de interpretación de los resultados se aplicó como punto de corte el valor del promedio obtenido por la muestra más una $\mathrm{DE}$, lo que significa que los pacientes con un puntaje mayor o igual a 76,8 perciben a su cuidador altamente sobrecargado con su cuidado.
- El tercer instrumento aplicado a los pacientes fue la escala de depresión del Centro de Estudios Epidemiológicos (CES-D, por sus siglas en inglés) de Estados Unidos, la cual permite detectar síntomas depresivos pero no establece un diagnóstico de esta patología, y ha sido validada en varios idiomas con un alpha de Crombach de entre 0,80 y 0,90 , aunque en el presente estudio fue de 0,748 (14). Sus resultados se interpretan de modo que a mayor puntaje mayor frecuencia de síntomas depresivos, con un mínimo posible de 0 y un máximo de 80 . Como punto de corte se usó el original de la escala que es de 16 puntos y fue establecido por Raldoff, basándose en el índice inferior del quintil superior de puntuaciones de la población general.

\section{Instrumentos aplicados a los cuidadores} principales:

- A los cuidadores se les aplicó la "escala de severidad de la fatiga" de Krupp y colaboradores, la cual ha sido utilizada como método de evaluación de fatiga en esclerosis múltiple y en otras condiciones de enfermedad crónica (15). Este instrumento ayuda asimismo a distinguir la fatiga de la depresión clínica, ya que ambas condiciones comparten síntomas. Con un alpha de Crombach de 0,84 obtenido en otros trabajos, en el presente alcanzó 0,789 (2). Con fines de interpretación de los resultados, se adjudica mayor puntaje a mayor sentimiento de fatiga, con un mínimo posible de 7 y un máximo de 63. Dado que se trata de una escala de puntaje continuo, como punto de corte se estableció el valor del promedio obtenido por la muestra más una $\mathrm{DE}$, lo que significa que los cuidadores con un puntaje mayor o igual a 44,5 se perciben a sí mismos fatigados.

- También se utilizó la CES-D con los cuidadores principales, en la misma versión aplicada a los pacientes (2).

La administración de los instrumentos en los pacientes fue realizada por personal entrenado mediante entrevistas llevadas a cabo durante el procedimiento de HDC, mientras que en el caso de los cuidadores principales los instrumentos fueron auto-administrados.

Para el análisis de los datos se utilizó el programa SPSS versión 12.0. Se calcu- laron estadísticos de tendencia central, promedio, DE y mediana para determinar las características sociodemográficas de pacientes y cuidadores principales. Para establecer la asociación entre algunas de las variables se utilizó la correlación de Pearson. Para determinar si existía diferencia entre grupos en alguna de las variables medidas se utilizó la $t$ de Student.

\section{RESULTADOS}

Del total de pacientes entrevistados, $40 \%$ fueron mujeres, con un promedio de edad de $56 \pm 14,9$ años. En 61,5\% de los casos la causa de la IRCT fue diabetes mellitus o hipertensión arterial. El promedio de comorbilidades fue de $2 \pm 1,2$ enfermedades, y el promedio de tiempo en HDC fue de $61 \pm 56,6$ meses, con una mediana de 48 meses.

En el caso de los cuidadores principales, $75 \%$ fueron mujeres con una edad promedio de $50 \pm 16,1$ años. Refirieron que estaban cuidando a su familiar desde hacía $59 \pm 54$ meses - con una mediana de 48 meses - y dedicando a esta actividad un promedio $18 \pm 8$ horas diarias. La totalidad de los cuidadores tenían algún lazo de consanguinidad con el paciente —en su mayoría, cónyuges. En el cuadro 1 se presentan los puntajes obtenidos por los pacientes y los cuidadores principales en los distintos instrumentos administrados.

Ahora bien, en la escala de autopercepción de carga 20,37\% (intervalo de confianza de 95\% [IC95\%]; 14,98-27,22) de los pacientes se ubicó sobre el punto de corte (76 puntos), lo que significa que percibieron que sus cuidadores estaban sobrecargados con su cuidado. Con respecto a la escala de apoyo social percibido, 16,67\% (IC95\%; 11,46-23,51) percibió un bajo apoyo social. Asimismo, $40,74 \%$ (IC95\%; 33,47-48,44) de los pacientes presentó síntomas depresivos en algún grado (CES-D), con mayor frecuencia en las mujeres $(t=3,826, P<0,01)$.

En relación con los cuidadores principales, 17,28\% (IC95\%; 12,23-23,84) se localizó sobre el punto de corte en la escala de severidad de la fatiga, lo que implica que se autopercibieron fatigados. Al discriminar por sexo, se encontró que el grado de fatiga era mayor en las mujeres, aunque con una diferencia no significativa. En la escala CES-D, 43,82\% (IC95\%; 36,42-51,53) de los cuidadores obtuvo un puntaje igual o mayor al punto de corte, 
CUADRO 1. Puntajes obtenidos en los instrumentos administrados para estudiar depresión y autopercepción de carga por parte de pacientes en hemodiálisis y de sus cuidadores, Chile, 2006

\begin{tabular}{lcccc}
\hline \multicolumn{1}{c}{ Instrumento } & Promedio & $\begin{array}{c}\text { Desviación } \\
\text { estándar }\end{array}$ & Mediana & Rango $^{\mathrm{a}}$ \\
\hline Paciente & & & & \\
$\quad$ Escala de autopercepción de carga & 52,80 & 23,99 & 52,5 & $8-100$ \\
$\quad$ Escala de percepción de apoyo social & 38,55 & 7,89 & 39,0 & $17-60$ \\
$\quad$ Escala de depresión del CES-D & 16,04 & 12,75 & 13,0 & $0-54$ \\
Cuidador principal & & & & \\
$\quad$ Escala de severidad de la fatiga & 29,39 & 15,11 & 28,0 & $9-63$ \\
Escala de depresión del CES-D & 16,63 & 12,04 & 13,0 & $0-45$ \\
\hline
\end{tabular}

Fuente: elaboración de las autoras con base en las referencias 7, 12 y 15.

a Puntaje mínimo y máximo obtenido en cada escala.

${ }^{\mathrm{b}}$ Centro de Estudios Epidemiológicos de Estados Unidos.

lo cual significa que presentaron algún grado de síntomas depresivos.

En cuanto a la asociación entre variables, se halló una asociación positiva $(r=0,43 ; P<0,001)$ entre la autopercepción de ser una carga y la presencia de síntomas depresivos en los pacientes. Por otra parte, se encontró una asociación inversa, aunque no significativa, entre la autopercepción de ser una carga y el apoyo social que percibe de su cuidador $(r=-0,112 ; P>0,157)$. Asimismo, se observó una asociación débil entre los años de cuidado y la fatiga del cuidador $(r=0,105 ; P=0,182)$.

\section{DISCUSIÓN}

Los cuidadores principales son por lo común mujeres y familiares, principalmente cónyuges, lo que es concordante con otros estudios realizados en pacientes en hemodiálisis $(16,17)$. La frecuencia de síntomas depresivos observada en los pacientes en HDC $(40,74 \%)$ y en los cuidadores principales $(43,82 \%)$ fue muy superior a la registrada en adultos chilenos por la Encuesta Nacional de Salud 2009-2010, donde apenas 17,2\% de la población general reveló dichos síntomas (18). En el caso de los pacientes en HDC, se sabe que los síntomas depresivos son frecuentes (50\% ó más los presentan) y que pueden afectar, entre otros, la adherencia a la terapia y el estado nutritivo $(19,20)$. Asimismo, la depresión es la complicación psicológica más frecuente en estos pacientes y puede aumentar la mortalidad (21).

Por otra parte, en el caso de los cuidadores principales hay evidencias de que el cuidado de otra persona se asocia con dificultades emocionales que incluyen depresión y una variedad de síntomas relacionados con la ansiedad (22). Ade- más, dado que la mayoría de los cuidadores principales son cónyuges, los síntomas depresivos se podrían explicar porque los roles maritales se ven alterados cuando uno de los esposos desarrolla una enfermedad crónica. Los esposos, al convertirse en cuidadores, pueden desarrollar sentimientos de hostilidad o depresión (23). Por otra parte, hay estudios que han demostrado que el ajuste marital es más débil en pacientes en hemodiálisis que en la población general. Este problema se presenta más en pacientes hombres, jóvenes y con altos niveles de educación (24). La literatura también informa que la demanda que se produce en el cuidador principal al asumir el cuidado de una persona con IRCT provoca en ellos conductas de negligencia hacia su propia salud, llevándolos con el tiempo a padecer fatiga (24).

No obstante, en el presente trabajo apenas $17,3 \%$ de los cuidadores se percibió a sí mismo fatigado, mientras que 20,3\% de las personas sometidas a HDC percibían que sus cuidadores estaban sometidos a sobrecarga como consecuencia de su cuidado. Esto podría explicarse porque los pacientes con IRCT en HDC están expuestos a múltiples estresores generados por su enfermedad, lo que en cierta manera afectaría sus relaciones más cercanas, familiares, sociales, culturales entre otras (23). Por otra parte, la autopercepción de carga se relacionó directamente con los síntomas depresivos que presentan los pacientes en HDC. El hecho de que una persona que cursa una enfermedad crónica sienta que su cuidador se encuentra sobrecargado por tener que atenderlo influye en la interacción entre ellos, produciendo ansiedad y depresión en la persona enferma con resultados negativos incluso en la adherencia a los tratamientos y controles (7).
Por lo mismo es importante que los profesionales de los centros de diálisis evalúen, aparte de los parámetros bioquímicos, los aspectos psicosociales de los pacientes con el fin de identificar problemas y abordarlos precozmente. Un ejemplo son los síntomas depresivos, ya que hay estudios que muestran que este trastorno influye en los resultados médicos de estos pacientes a raíz de cambios en el sistema inmunológico, en la respuesta al estrés, en su estado nutricional y en la adherencia al tratamiento (23). También en los cuidadores es preciso prestar atención a la presencia de cualquier signo de alteraciones psicosociales - como fatiga o depresión-con objeto de identificarlas y tratarlas lo antes posible.

Este estudio tiene algunas limitaciones que es importante explicitar. Una de ellas es que se administró el mini-examen del estado mental de Folstein a los pacientes, pero no a los cuidadores principales, lo cual podría haber introducido un sesgo considerando que algunos de ellos eran adultos mayores. Otra radica en que la aplicación de los instrumentos a los pacientes tuvo lugar durante la sesión de HDC, factor que pudo afectar la privacidad durante la entrevista y aumentar la deseabilidad social en las respuestas. Sin embargo, esta es una práctica habitual, dada la cantidad de tiempo que pasan los pacientes en los CD.

Se notó que la asociación entre percepción de alta carga del cuidador por parte del paciente y bajo apoyo social percibido puede derivar - entre otros efectos negativos- en un aislamiento del paciente, observación que debería ser confirmada en estudios futuros dado que en el presente trabajo no alcanzó significación estadística. En todo caso, esta asociación inversa no es extraña si se recuerda que la escala de autopercepción de carga mide en forma muy general la percepción que tiene el paciente de lo que implica su cuidado para el cuidador —es decir cuánta carga representa el cuidado instrumental del paciente (p. ej. ayudarlo a seguir la dieta y acompañarlo a los controles médicos); en cambio, la escala de apoyo social percibido utilizada mide principalmente la percepción del paciente de contar con apoyo emocional en general, sea del cuidador, de familiares, de amigos $\mathrm{u}$ otros significativos. En estudios futuros se recomienda asimismo incorporar la percepción del cuidador con respecto a su carga y determinar si se asocia positivamente con la percepción del paciente. 
Adicionalmente, debido a que el presente estudio halló que los síntomas de depresión se encuentran presentes tanto en los pacientes como en los cuidadores, se recomienda aplicar la CES-D en forma sistemática a la díada pacientecuidador con el fin de referir, para confirmación de diagnóstico y, si fuera necesario, tratamiento a los casos que puntúen alto en este instrumento. Por otro lado, el equipo de salud de los CD debería incorporar intervenciones dirigidas a pacientes y cuidadores con el objeto de prevenir y detectar síntomas depresivos.

Quedó claro que tanto los pacientes en HDC como sus cuidadores presentan síntomas depresivos en un porcentaje importante, que la mayoría de los pa- cientes no perciben sobrecargados a sus cuidadores principales y reportan un apoyo social moderado o alto, y que la mayoría de los cuidadores no se perciben a sí mismos fatigados. Se encontró además una asociación estadísticamente significativa entre autopercepción de carga y presencia de síntomas depresivos en los pacientes, y que no hay asociación entre autopercepción de carga y apoyo social en personas sometidas a HDC, ni entre los años de cuidado y la fatiga del cuidador. Estos hallazgos reafirman la relevancia de brindar respaldo psicosocial o asesoramiento tanto al paciente como a su cuidador, servicios que en primera instancia deberían estar a cargo de enfermeras y médicos de los CD entrenados para ofrecerlos. Más aún, haría falta evaluar sistemáticamente en este grupo la presencia de síntomas de depresión con objeto de detectarlos y referir el caso a tiempo a un profesional especializado.

Por último, sería de gran utilidad para los pacientes contar con una enfermera de cabecera que conozca en profundidad cada caso de modo que pueda detectar tempranamente síntomas de depresión o de aislamiento social. Dada su posición ideal para asistir a los pacientes y los cuidadores porque pueden observar el impacto de la terapia dialítica en el día a día de las familias, estas profesionales podrían proporcionar apoyo emocional e informacional para adaptarse a la incertidumbre que significa vivir con una falla renal crónica.

\section{REFERENCIAS}

1. Smith SR, Soliday E. The effects of parental chronic disease on the family. Fam Relat. 2001;50(2):171-7.

2. Schneider RA. Fatigue Among Caregivers of Chronic Renal Failure Patients: A Principal Components Análisis. Nephrol Nurs J. 2003; 30(6):629-33.

3. Tavallaii SA, Nemati E, Khoddami HR, Farahani MA, Lankarani MM, Assari S. Marital Adjustment in Patients on Long-Term Hemodialysis: A Case-Control Study. Iran J Kidney Dis. 2009;(3):156-61.

4. Hunt CK. Concepts in Caregiver Research. J Nurs Scholarsh. 2003;35(1):27-32.

5. Krespi MR, Bone M, Almad R, Worthington B, Salmon P. Hemodialysis patients' evaluation of their lives. Turk Psikiyatri Derg. 2008; 19(4):365-72.

6. Kimmel PL, Peterson RA, Weihs KL, Simmens SJ, Alleyne S, Cruz I, et al. Psychosocial factors, behavioral compliance and survival in urban hemodialysis patients. Kidney Int. 1998;54(1):245-54.

7. Cousineau N, McDowell I, Hotz S, Hébert P. Measuring chronic patients' feelings of being a burden to their caregivers: development and preliminary validation of a scale. Med Care. 2003;41(1):110-8.

8. Poblete H. XXIII Cuenta de hemodiálisis crónica (HDC) en Chile. Sociedad Chilena de $\mathrm{Ne}-$ frología. Registro de diálisis. 2003;23:1-100.

9. Tilden VP, Weinert C. Social Support and the Chronically Ill Individual. Nurs Clin North Am. 1987;22(3):613-21.

10. Thoits PA. Stress, coping and social support processes: Where are we? What next? J Health Soc Behav. 1995; Spec No:53-79.
11. Venegas BB. Habilidad del cuidador y funcionalidad de la persona cuidada. Aquichan. 2006;6(1):137-47.

12. Zimet GD, Dahlem NW, Zimet SG, Farley GK. The multidimensional scale of perceived social support. J Pers Assess. 1988;52(1):30-41.

13. Arechabala MC, Miranda CC. Validación de una escala de apoyo social percibido por un grupo de adultos mayores adscritos a un programa de hipertensión de la región metropolitana. Cienc Enferm. Disponible en: http:// www.scielo.cl/scielo.php?script=sci_arttext \&pid=S0717-95532002000100007\&lng=es. Acceso el 14 de mayo de 2011.

14. Gempp R, Thieme C. Efecto de diferentes métodos de puntuación sobre la fiabilidad, validez y puntos de corte de la escala de depresión del Centro para Estudios Epidemiológicos (CES-D). Terapia psicológica. Disponible en: http://www.scielo.cl/pdf/terpsicol/ v28n1/art01.pdf. Acceso el 14 de mayo de 2011.

15. Krupp LB, LaRocca NG, Muir Nash J, Steinberg AD. The Fatigue severity scale. Application to patients with multiple sclerosis and systemic lupus erythematosus. Arch Neurol. 1989;46:1121-3.

16. Chii JC, Hsing-Yi C, Pin C, Hsiu HW. Social support and caregiving circumstances as predictors of caregiver burden in Taiwan. Arch Gerontol Geriatr. 2009;48:419-24.

17. Weety S, Ching L. The home care experience as perceived by the caregivers of Chinese dialysis patients. Int J Nurs Stud. 2002;39(3):269-77.

18. Ministerio de Salud, Chile. Santiago: Encuesta Nacional de Salud. Gobierno de Chile, 20092010. Disponible en: http://www.redsalud. gov.cl/portal/url/item/99c12b89738d80d5e0 4001011e0113f8.pdf. Acceso el 14 de mayo de 2011.

19. García FW, Fajardo C, Guevara R, Gonzáles V, Hurtado A. Mala adherencia a la dieta en hemodiálisis: papel de los síntomas ansiosos y depresivos. Nefrologia. 2002;22(3):245-52.

20. Alvarez-Ude F, Fernadez MJ, Vásquez A, Mon C, Sánchez R, Rebollo P. Síntomas físicos $\mathrm{y}$ trastornos emocionales en pacientes en programa de hemodiálisis crónica. Nefrologia. 2001;21(2):191-9.

21. Koo JR, Yoon JW, Kim SG, Lee YK, Oh KH, $\mathrm{Kim} \mathrm{GH}$, et al. Association of depression with malnutrition in chronic hemodialysis patients. Am J Kidney Dis. 2003;41(5):1037-42.

22. Gayomali $\mathrm{Ch}$, Sutherland S, Finkelstein F. The challenge for the caregiver of the patient with chronic kidney disease. Nephrol Dial Transpl. 2008;23(12):3749-51.

23. Belasco AG, Sesso R. Burden and quality of life caregivers for hemodialysis patients. Am J Kidney Dis. 2002;39(4):805-12.

24. Cukor D, Cohen S, Peterson R, Kimmel P. Psychosocial aspects of chronic disease: ESRD as a paradigmatic illness. J Am Soc Nephrol. 2007;18:3042-55.

Manuscrito recibido el 30 de septiembre de 2010. Aceptado para publicación, tras revisión, el 4 de abril de 2011. 
ABSTRACT Objective. To determine the depressive symptoms, perceived social support, and self-perceived burden in chronic hemodialysis (CHD) patients; identify depressive symptoms and degree of fatigue in caregivers of CHD patients; evaluate-in pa-

Depression and self-perceived burden of care by hemodialysis patients and their caregivers tients-the association between self-perceived burden and perceived social support, and between self-perceived burden and depressive symptoms; and evaluate-in caregivers - the relationship between the number of years of care and the degree of fatigue reached.

Methods. A sample of 162 patient-caregiver pairs was selected in which an adapted version of the Multidimensional Scale of Perceived Social Support, along with the Self-perceived Burden Scale, the Center for Epidemiologic Studies Depression Scale, and the Fatigue Severity Scale were applied. The instruments were administered to patients by trained research assistants and, in the case of the caregivers, were self-administered.

Results. Forty percent of the patients were women, with an average age of $56 \pm 14.9$ years, whereas $75 \%$ of the primary caregivers were female spouses, with an average age of $50 \pm 16.1$ years. It was observed that the patients $(40.74 \%)$ as well as the primary caregivers $(43.82 \%)$ had depressive symptoms. A positive association $(r=0.43$, $P<0.001)$ was found between self-perceived burden and the presence of depressive symptoms in patients.

Conclusions. The majority of the primary caregivers were women and relatives of the patients. They perceived themselves as fatigued with care, as did the patients themselves. The presence of depressive symptoms was similar in patients and caregivers. There was a statistically significant association between self-perceived burden and the presence of depressive symptoms in patients.

Key words Hemodialysis; depressive symptoms; chronic disease; mental health; Chile. 